\title{
Diabetic autonomic neuropathy after pancreas and kidney transplantation
}

\author{
P. Bouček, V. Bartoš, I. Vaněk, Z. Hýža and J. Skibová \\ Institute for Clinical and Experimental Medicine, Prague, Czechslovakia
}

Summary. We have evaluated the effect of successful pancreatic and kidney transplantation on autonomic neuropathy in nine Type 1 (insulin-dependent) diabetic subjects. Cardiovascular reflex tests were performed before and at 6-24 months after transplantation. A control group of ten Type 1 diabetic patients after kidney grafting only was examined at the same time periods. For base-line comparisons results of the tests in ten healthy subjects were used. Advanced autonomic neuropathy was present in both groups of transplant recipients and no significant changes in test results could be found at post-transplant evaluation. Lack of autonomic nerve function improvement was confirmed in seven patients of the pancreatic and kidney transplantation group who were examined again after 2-4 years. Irreversible structural autonomic nerve damage is probably present in uraemic pancreatic transplantation candidates.

Key words: Pancreatic transplantation - Autonomic neuropathy - Cardiovascular function tests

\section{Introduction}

Autonomic nervous system dysfunction is a common complication of long-standing diabetes mellitus, although clinical symptoms are rarely present (Watkins 1990; Bilous 1990). Autonomic neuropathy can be found almost universally in patients with overt diabetic nephropathy and its severity progresses with the development of renal insufficiency (Zander et al. 1989; Heidbreder et al. 1985). While most parts of the autonomic nervous system may be affected, it is the cardiovascular, gastrointestinal and genito-urinary damage that can result in severe and even life-threatening consequences. Attempts to reverse the progressive course of diabetic autonomic neuropathy have included pharmacological intervention with aldose reductase inhibitors or gangliosides, as well as trials establishing near-normoglycaemia with the use of intensive insulin regimens. With a few exceptions the results have been mostly disappointing, although prolonged experience with treament is scarce (Watkins 1990; Bilous 1990). Long-term normoglycaemia can be effectively established in diabetic subjects after pancreatic transplantation and the influence of this procedure on the course of diabetic complications is being studied extensively. We have evaluated the effect of successful pancreatic transplantation on advanced autonomic neuropathy in Type 1 diabetic patients.

\section{Subjects and methods}

Nine Type 1 diabetic patients (three male and six female, mean $( \pm S D)$ age $36.8 \pm 7.4$ years, mean duration of diabetes $23.8 \pm$ 7.1 years) with end-stage diabetic nephropathy, who underwent a successful combined pancreatic and kidney transplantion between June 1985 and August 1989, were included in the study (Group TXPK). The control group comprised ten Type 1 diabetic patients (seven male and three female, mean age $36.7 \pm 4.6$ years, mean duration of diabetes $19.2 \pm 4.7$ years) who received between June 1984 and February 1990 a kidney graft only (Group TXK). A group of ten healthy subjects (eight male and two female, mean age $39.3 \pm 10.9$ years) was also used for the comparison of pre-transplant results of autonomic nervous system function assessment. All groups were comparable with respect to age $(p>0.05)$. There was no significant difference in the mean duration of diabetes, pre-transplant daily insulin dose (TXPK vs TXK $42 \pm 7$ vs $45 \pm 8 \mathrm{IU}, \mathrm{p}>0.05$ ) and $\mathrm{HbA} 1 \mathrm{c}$ level (TXPK vs TXK $11.1 \pm 1.5$ vs $9.7 \pm 1.9 \%$, p $>0.05$, upper limit of normal values $6.1 \%$ ) in both groups of patients.

Segmental duct-occluded pancreatic grafts and kidneys from cadaveric donors were anastomosed to iliac vessels. A triple immunosuppressive regimen (cyclosporine A, azathioprine and prednisone) was used in all patients. All pancreatic graft recipients were free from insulin injections during the 

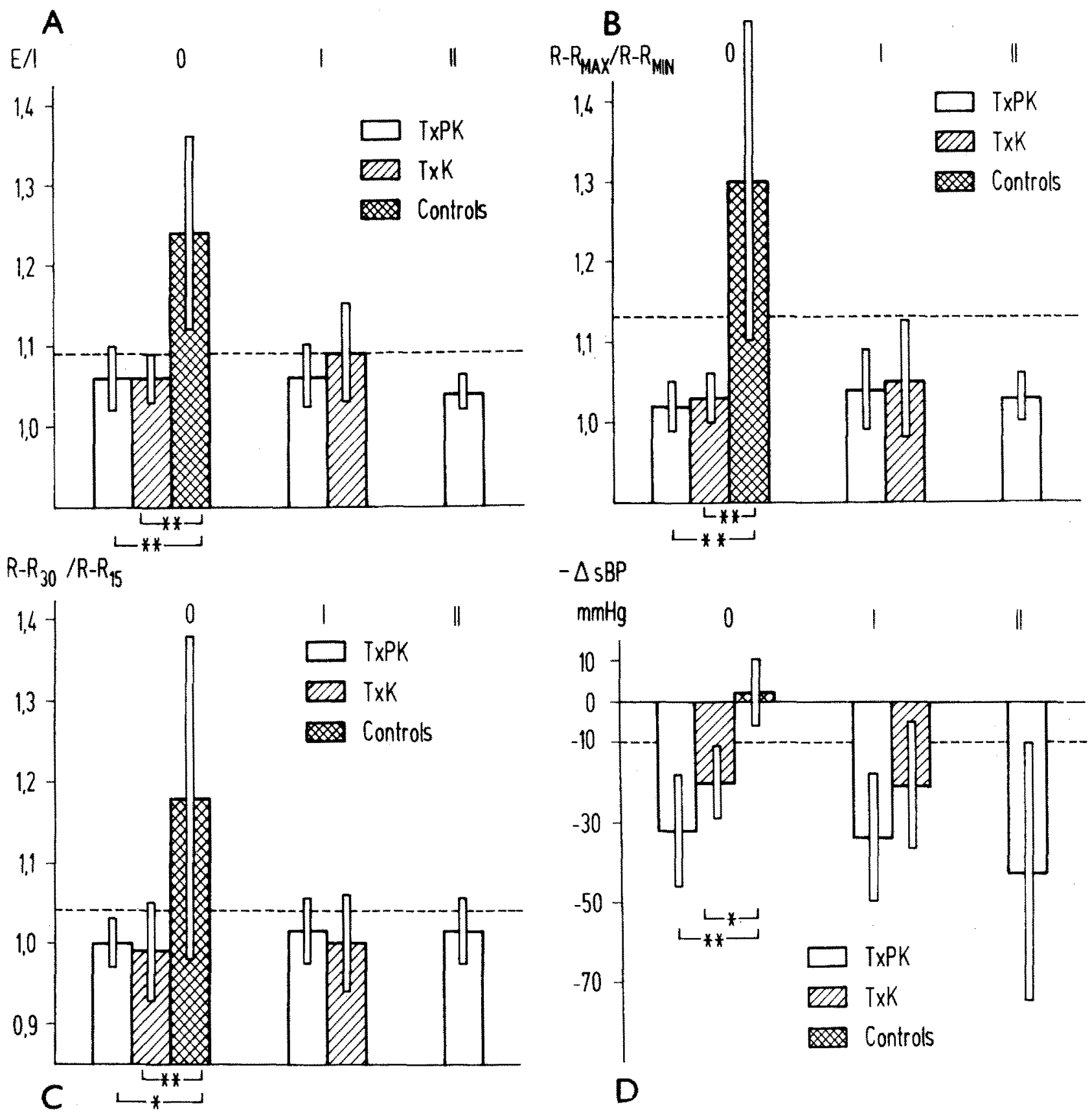

post-transplant period while recipients of a kidney graft alone were treated with continuous subcutaneous insulin infusion (nine patients) or multiple daily insulin injections (one patient). The mean serum-creatinine level was comparable in both patient groups after transplantation (TXPK vs TXK $112 \pm$ 24 vs $122 \pm 23 \mathrm{umol} / \mathrm{l}, \mathrm{p}>0.05$ ). The mean $\mathrm{HbA}_{1} \mathrm{C}$ post-transplant level was slightly but not significantly higher in the TXK group (TXPK vs TXK $5.9 \pm 1.7$ vs $6.5 \pm 1.9 \%$, $\mathrm{p}>0.05$ ).

Fig.1 (A-D) Results of cardiovascular function tests in pancreas and kidney recipients (TXPK), recipients of kidney alone (TXK) and healthy subjects (control subjects). (A) Heart rate variability with deep breathing; ( $B$ and $C$ ) heart rate response to orthostasis; (D) systolic blood pressure response to orthostasis. $0=$ pre-transplant, $\mathrm{I}=2-24$ months and $I I=$ 2-4 years post-transplant. Mean \pm SD. Interrupted line indicates lower limit of normal and borderline values from Sundkvist and Lilja (1985), Ewing et al. (1985) and Wieling et al. (1982). 
Standard cardiovascular function tests were used for the evaluation of autonomic neuropathy. The heart rate (as electrocardiogram (ECG) R-R interval) variability induced by deep breathing during $1 \mathrm{~min}$ at 6 breaths per $\min$ was expressed as the expiration/inspiration (E/I) ratio (Sundkvist and Lilja 1985). Heart rate reaction to orthostasis was assessed by the ratio of the 30th to the 15 th ECG $R-R$ interval (R-R $30 / R-R_{15}$ ) (Ewing et al. 1985) and also of the maximal to minimal ECG $R-R$ interval ( $R-R_{\max } / R-R_{\min }$ ) (Wieling et al. 1982) after standing up. The ECG $R-R$ intervals were monitored throughout the procedure and fed into a computer for the calculation of the indices. Blood pressure reaction to orthostasis was measured by an indirect oscillometric method. The maximal value of systolic blood pressure fall during the first $3 \mathrm{~min}$ after standing up ( $\triangle \mathrm{sBP}$ ) was used (Ewing et al. 1985). All patients were investigated in the pre-transplant period and again at 6-24 months post-transplantation. The investigations were repeated at 2-4 years in seven patients of the pancreatic transplant group.

\section{Statistical analysis}

As the results did not follow a normal distribution we used the Kruskal-Wallis and Wilcoxon rank-sum tests for comparisons between groups. For the comparison of results within a group over time the Wilcoxon signed rank test was used. All tests were two-tailed. P values of less than 0.05 were considered as significant.

\section{Results}

Abnormal results of all tests of autonomic function were found at base-line investigation in both groups of transplanted patients when compared to the healthy subjects (Fig. 1, A-D). The mean results within both groups were well below the lower limits of normal and borderline values as previously published (Sundkvist and Lilja 1985; Ewing et al. 1985; Wieling et al. 1982). We found no significant differences between the two groups of transplant recipients in any of the tests.

None of the test results in either of the patient groups improved at 6-24 months after transplantation (there was a small increase in the E/I value in the TXK group, not however reaching significance, $(\mathrm{p}=0.07)$. Again, there was no significant difference in any of the tests when comparing the two groups of transplant patients. The repeated examination of pancreatic graft recipients (seven patients) at 2-4 years after transplantion also revealed no significant changes in any of the tests. Figure 2 shows the evolution of the $\mathrm{E} / \mathrm{I}$ index (heart rate variability with deep breathing) values in individual patients. No clear trend is discernible from these results. The lower limit of normal and borderline values was not reached by any patient.

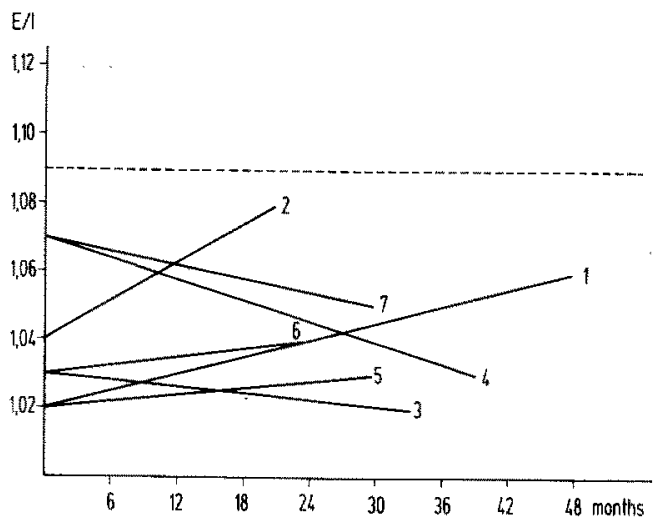

Fig.2 Base-line and last results of the test of heart rate variability with deep breathing in pancreas and kidney recipients. Interrupted line indicates lower limit of normal and borderline values from Sundkvist and Lilja (1985).

\section{Discussion}

The fate of diabetic neuropathy after successful pancreatic transplantation has been studied by a few groups recently (Schafferhans et al. 1986; Solders et al. 1987; Kennedy et al. 1990; Nusser et al. 1990). Careful analysis with the use of several clinical and neurophysiological parameters has shown some amelioration of peripheral nerve function such as increases in conduction velocities (Solders et al. 1987; Kennedy et al. 1990). On the other hand, these studies have failed to demonstrate a clear improvement in autonomic neuropathy as assessed by cardiovascular function tests (Schafferhans et al. 1986; Solders et al. 1987). Nevertheless, in two studies from the Minneapolis and Munich transplant centres including the largest numbers of patients so far, at least deterioration of autonomic nerve function could be prevented in pancreatic graft recipients (Kennedy et al. 1990; Nusser et al. 1990).

Similar to other groups we have chosen cardiovascular reflex tests for the evaluation of diabetic autonomic neuropathy in patients transplanted at our centre. In comparison to diagnostic procedures employed for this purpose in other organ systems, these tests are simple to carry out, non-invasive and sufficiently reliable to enable a longitudinal follow-up (Ewing et al. 1985). As in every assessment of the autonomic nervous system function, meticulous attention must be paid to the standardisation of the procedures. This raises some questions concerning the possible influence of immunosuppressive, and possibly also other (e.g. antihypertensive) therapy, when comparing pre- and post-transplantation results within the studied group.

The course of clinical autonomic dysfunction has not been followed in a standardised manner in our patients. The pathogenesis of some of the clinical symptoms and 
signs is probably multifactorial and their severity sometimes varies considerably even in the natural history of diabetes (e.g. diarrhoea). A trend toward improvement of the subjective symptomatology was noted among our patients in the context of a general amelioration of the quality of life.

The lack of improvement in autonomic nervous system function, which was confirmed in our study is probably caused by extensive structural damage to the nerves concerned. A hypothetical difference of the capacity for repair of various nerve fibre types may offer an explanation for the slight amelioration of peripheral nerve function reported in other studies. While the number of pancreas graft recipients evaluated in this trial is certainly insufficient to preclude a type 2 statistical error, we are less convinced than other groups, that longer periods of follow-up may produce a measurable improvement. Nevertheless, progression of autonomic dysfunction may be retarded or even halted in patients who had undergone transplant surgery in earlier stages of diabetic nephropathy. Such patients formed a substantial part of the group investigated at the Minneapolis centre (Kennedy et al. 1990).

Thus, cardiovascular autonomic neuropathy, which has been recognised as an independent risk factor in diabetic patients (Watkins 1990; Bilous 1990), is not clearly reversed by successful pancreatic transplantation. However, interestingly, Navarro et al. (1990) have shown in a recent study that among patients with abnormal autonomic function, survival rates were better in those with a functioning pancreatic transplant.

\section{References}

Bilous RW (1990) Diabetic autonomic neuropathy. A common complication which rarely causes symptoms. $\mathrm{Br}$ Med J 301:565-567

Ewing DJ, Martyn CN, Young RJ, Clarke BF (1985) The value of cardiovascular autonomic function tests: 10 years experience in diabetes. Diabetes Care 8:491-498

Heidbreder E, Schafferhans K, Heidland A (1985) Autonomic neuropathy in chronic renal insufficiency. Comparative analysis of diabetic and nondiabetic patients. Nephron 41:50-56

Kennedy WR, Navarro X, Goetz FC, Sutherland DER, Najarian JS (1990) Effects of pancreatic transplantation on diabetic neuropathy. N Engl J Med 322:1031-1037

Navarro X, Kennedy WR, Loewenson RB, Sutherland DER (1990) Influence of pancreas transplantation on cardiovascular relexes, nerve conduction, and mortality in diabetes mellitus. Diabetes 39:802-806

Nusser J, Kehrle M, Scheuer R, Illner W, Abendroth D, Land W, Landgraf R (1990) Follow-up of autonomic neuropathy after pancreas and/or kidney transplantation. Diabetologia 33(Suppl.) :34 (Abstract)
Schafferhans K, Heidbreder E, Land W, Walter U, Stürmer W, Götz R, Heidland A (1986) Diabetic autonomic neuropathy after simultaneous transplantation: progressively across the Rubicon? Transplant Proc 18:1136-1138

Solders G, Gunnarsson R, Persson A, Wilczek H, Tydén G, Groth C-G (1987) Effects of combined pancreatic and renal transplantation on diabetic neuropathy: a two-year follow-up study. Lancet 2:1232-1235

Sundkvist G, Lilja B (1985) Autonomic neuropathy in diabetes mellitus: a follow-up study. Diabetes Care 8:129-133

Watkins PJ (1990) Diabetic autonomic neuropathy. N Engl J Med 322:1078-1079

Wieling W, van Brederode JFM, de Rijk LG, Borst C, Dunning AJ (1982) Reflex control of heart rate in normal subjects in relation to age: a data base for cardiac vagal neuropathy. Diabetologia 22:163-166

Zander E, Schulz B, Heinke P, Grimmberger E, Zander G, Gottschling HD (1989) Importance of cardiovascular autonomic dysfunction in IDDM subjects with diabetic nephropathy. Diabetes Care 12:259-264

Dr. P. Boucek

Institute for Clinical and Experimental Medicine

Department of Medicine I

Vídenská 800

14000 Prague 4

Czechoslovakia 